\title{
Case analysis of early COVID-19 complicated with PLT abnormality
}

\author{
Youlai Zhang ${ }^{1}$, Guohua Xin ${ }^{2}$, Lijin Zou², Zhongyuan Yin ${ }^{2}$, and Yuanlin Zeng ${ }^{2}$ \\ ${ }^{1}$ First Affiliated Hospital of Nanchang University \\ ${ }^{2}$ Affiliation not available
}

November 2, 2020

\begin{abstract}
Objective: To investigate the causes of early COVID-19 complicated with platelets(PLT) abnormality, and to analyze the possible mechanisms. Methods: The case datum of early COVID-19 complicated with PLT increase or decrease was collected. $(125-350) \times 109 / \mathrm{L}$ defined as the normal level of PLT(Group C), $<125 \times 109 / \mathrm{L}$ was defined as the PLT decrease group (Group A), $>350 \times 109 /$ L defined as the PLT increase group (Group B). The data were analyzed after collected. Results: Our statistical results showed that the incidence of COVID-19 combined PLT decreased was about $11.94 \%$ and the incidence of combined PLT increased was about $9.33 \%$ at admission. Compared with Group B and C, Group A showed a significant decrease in white blood cell, neutrophil and CD4 $(\mathrm{P}<0.05)$. The lymphocyte in Group A and C decreased significantly, but not find in Group B $(\mathrm{P}<0.05)$. Compared with Group A and C, IL-4 was increased in Group B, but lymphocyte decline was not significant $(\mathrm{P}>0.05)$. Conclusion: PLT abnormalities occur in all patients with different types of COVID-19. It may be related to the severity of inflammation and infection, immune regulation and megakaryocyte function, etc.
\end{abstract}

\section{Case analysis of early COVID-19 complicated with PLT abnormality}

Zhang Youlai ${ }^{1}$, Xin Guohua ${ }^{1}$, Zou Lijin ${ }^{1}$, Yin Zhongyuan ${ }^{3}$, Zeng Yuanlin ${ }^{1}$,

1.Burn Center, The First Affiliated Hospital of Nanchang University, Nanchang, Jiangxi, 330006, China

2. Cancer center, Tongji Medical College, Huazhong University of science and technology, Wuhan, Hubei Province, 430000, China

Corresponding author:Zeng Yuanlin, Burn Centre, The First Affiliated Hospital of Nanchang University, 17 Yongwai Street, Nanchang, 330006.

E-mail:1504133139@qq.com

\section{Abstract:}

Objective : To investigate the causes of early COVID-19 complicated with platelets(PLT) abnormality, and to analyze the possible mechanisms.

Methods: The case datum of early COVID-19 complicated with PLT increase or decrease was collected. $(125-350) \times 10^{9} /$ L defined as the normal level of PLT(Group C), $<125 \times 10^{9} / \mathrm{L}$ was defined as the PLT decrease group (Group A), $>350 \times 10^{9} / \mathrm{L}$ defined as the PLT increase group (Group B) . The data were analyzed after collected.

Results: Our statistical results showed that the incidence of COVID-19 combined PLT decreased was about $11.94 \%$ and the incidence of combined PLT increased was about $9.33 \%$ at admission. Compared with Group $\mathrm{B}$ and $\mathrm{C}$, Group A showed a significant decrease in white blood cell, neutrophil and $\mathrm{CD}_{4}(\mathrm{P}<0.05)$. The 
lymphocyte in Group A and C decreased significantly, but not find in Group B $(\mathrm{P}<0.05)$. Compared with Group A and C, IL-4 was increased in Group B, but lymphocyte decline was not significant $(\mathrm{P}>0.05)$.

Conclusion: PLT abnormalities occur in all patients with different types of COVID-19. It may be related to the severity of inflammation and infection, immune regulation and megakaryocyte function, etc.

Keywords: COVID-19, PLT, Megakaryocyte

The interaction between PLT and endothelial cells has been shown to play a role in the formation of acute lung injury caused by influenza virus(Rommel et al.,2019). Study have reported that PLT reduction in COVID-19 patient(Huang et al.,2020). We found that PLT was not only decreased, but also increased in many COVID-19 patients. In this paper, COVID-19 complicated with PLT abnormality cases at admission were collected and analyzed.

\section{Research objects and test methods}

Retrospective study COVID-19 patients are admitted to the Tumor Center affiliated to Tongji Medical College of Huazhong University of Science and Technology. PLT abnormality case datum was gathered. Indicators including WBC, RBC, hemoglobin, neutrophils, lymphocytes and T lymphocyte subsets, cytokines (IL2, 4, 6, 8, 10, TNF - $\alpha$, IFN - $\gamma$ ), CRP and PCT. and according to the PLT clinical reference range divide 3 groups, (125-350) $\times 10^{9} / \mathrm{L}$ defined as the normal level of PLT(Group C), $<125 \times 10^{9} / \mathrm{L}$ was defined as the PLT decrease group (Group A), $>350 \times 10^{9} /$ L defined as the PLT increase group (Group B). Comparing the above indicators between each group. Exclusion criteria: prior to COVID-19, there were underlying diseases or drugs that caused PLT abnormalities, pregnant women,and so on.

COVID-19 diagnostic criteria: Refer to the COVID-19 Diagnosis and Treatment Protocol (Trial version 6). Novel Coronavirus nucleic acid positive(test by real-time FLUORESCENCE RT-PCR). Clinical classification: critical, severe and normal (including light in article)type. All patients were treated in the same way after admission, including regular CT, nucleic acid test, oral antiviral, anti-inflammatory,etc.

\section{Statistical methods}

Data were analyzed by SPSS22.0 software and checked by T-test, chi-square test or Fisher's exact probability test. $\mathrm{P}<0.05$ was considered statistically significant.

\section{Results}

3.1 In our study, we had investigated 268 COVID-19 patients and found 32 cases (11.94\%) PLT decreased patients at admission, including 22 males and 10 females. The age ranged from 29 to 84 years old. most of them were[?]60 years old. the PLT value were between (15-124 )x109/L, but only 8 cases were lower than $100 \times 10^{9} / \mathrm{L}$. Among them, 2 case were critically severe type, 16 were heavy type, and 14 were normal type. 25 patients ( $9.33 \%$ ) PLT increased been found at admission, including 16 males and 9 females. The age ranged from 23 to 83 years old, and the PLT value were between (351-541) x10 $/ \mathrm{L}$, of which only 1 case was critical heavy type, 13 cases were heavy type, and 11 cases were normal type.

3.2 There was no statistically significant difference between the three groups in terms of RBC and hemoglobin at admission $(\mathrm{P}>0.05)$, as shown in table 1. Compared with Group B and $\mathrm{C}$, there was a decrease in WBC in Group $\mathrm{A}(\mathrm{P}<0.05)$. The lymphocyte in Group $\mathrm{A}$ and $\mathrm{C}$ decreased significantly compared with Group $\mathrm{B}(\mathrm{P}<0.05)$. The absolute value/percentage of neutrophils and the percentage of lymphocytes were not significantly different $(\mathrm{P}>0.05)$, as shown in table 1-2.

3.3 Among the 57 patients with abnormal PLT, except for one in group A, the values of PCT of the other 56 patients were in the normal range; IFN- $\gamma$ was normal too; Only one case patient's NF- $\alpha$ was elevated in each group. There was no significant difference in the comparison of CD3, IL2, IL4, and IL10 among patients in the three groups at admission $(\mathrm{P}>0.05)$, but CD4 in Group A was lower than that in Group $\mathrm{B}$ and $\mathrm{C}(\mathrm{P}<0.05)$, as shown Table 3-4. IL-6 was increased in all patients, but the range of IL-6 values fluctuated widely. There were more cases of IL-6 value [?] $50 \mathrm{pg} / \mathrm{ml}$ in Group A, while B and C Group have 
no significant difference $(\mathrm{P}>0.05)$; $\mathrm{CRP}$ was not statistically significant in the number of cases $(\mathrm{P}>0.05)$, but values [?]50mg / L, Group A was more than Group B (P <0.05). IL-4 value in Group B was significantly higher than that in Group $\mathrm{C}(\mathrm{P}<0.05)$, but there was no significant difference between Group A and C.

\section{Discussion}

Clinical treatment has found that platelet abnormal are prone to occur during the course of COVID-19 treatment. Continuous low platelets can be employed as an indicator of no improvement in disease and poor prognosis. We found that many COVID-19 patients were concurrent with PLT decrease when admitted to hospital. Zhong Nanshan also reported that 36.2\% of COVID-19 patients had thrombocytopenia at admission(Huang et al.,2020). Early thrombocytopenia occurred in all types of COVID-19 patients, which were further confirmed in our study. However, the percentage of thrombocytopenia in our article is not as low as reported in this literature, except that our standard is lower (PLT $<125 \times 10^{9} / \mathrm{L}$, literature is $<150 \times 10^{9} / \mathrm{L}$ )(Huang et al.,2020). we analyze that On the one hand, it may be related to the length of the isolation period, and antiviral treatment has been given before the nucleic acid test is positive; On the other hand, The case data of our study be later than the reported data in literature, and Whether or not the virulence of new crown pneumonia virus has abated? It needs for further study.

In this study, it was found that PLT abnormalities of COVID-19 patients at admission may be less correlated with PCT, IL-2, IL-10, IFN- $\gamma$, TNF- $\alpha$, CD3, CD8, erythrocyte and hemoglobin. CD4/8, IL-6 and CRP were only difference compared with Group A and B,. IL-6 and CRP reflect the severity of inflammation and infection. The higher the value, the more severe the inflammation and the more sensitive the IL-6 response.

The literature confirms(Zhu,et al.,2014)that PLT is a repository of multiple inflammation and immunoregulatory factors, and act as "capable coordinators" in inflammation and immune responses. However, it is still unclear about the cause of thrombocytopenia after viral infection. There may be two mechanisms used to explain(Yang,et al.,2004). First, virus infection damages hematopoietic stem cells and indirectly inhibit hematopoietic cells through immune mechanisms. Second, lung is one organ of megakaryocytes ( MKs ) maturing and PLT releasing(Lefrançais et al.2017). Due to extensive lung injury, megakaryocyte division is reduced, and inflammation lead to PLT aggregation and thrombosis, which increase PLT consumption and reduce production. However, early lung injury is not particularly serious, and platelets can return to normal levels as the lung inflammation or infection continues to worsen during treatment. In addition, some patients also platelet elevation. So the above mechanism obviously does not seem to fully explain the early thrombocytopenia in COVID-19 patients.

Robert A. Campbell found that(Campbell,et al.,2019)MKs have the ability to fight viruses through the IFITM3 factor. PLT IFITM3 factor isolated from patients infected with dengue fever (DENV) or influenza virus is significantly rising. Low expression of IFITM3 in PLT is associated with severity of disease and increased mortality. DENV-infected human MKs can selectively up-regulate type I interferons (IFNs) and IFITM3. Overexpression of IFITM3 in MKs can effectively prevent DENV infection. Whether PLT and MKs can play the same role in COVID-19 patient has not been studied until now. So we think that if it can play the same role, It may be more reasonable to explain the early PLT reduction in COVID-19 patient than the above two possible mechanism. Because we found that most patients with early PLT reduction are "transient". We speculate that after the lung infection of the new coronavirus, a large number of early PLT and megakaryocytes were used to fight the virus instantly, resulting in a decrease of PLT, and the relatively slow response to inflammation, the platelet regulation mechanism gradually PLT returned to normal.

However. PLT was also found to be elevated in the early stage of COVID-19, which may be our first report. First of all. We consider whether there will be a difference in the virus incubation time between the PLT decreased and elevated group? . In this study, it was found that lymphocytes were less decreased in the PLT elevated group, and IL-4 was higher than that in the thrombocytopenia and PLT normal groups. In addition, the values of IL-6 and CRP in PLT elevated group, indicators of inflammation and infection, were also lower than those of the thrombocytopenia group, but were not different from those of the PLT normal group. Therefore, according to our analysis, it may be due to the different incubation time of the virus, the 
difference between drug control and individual patients, and the growth effect of PLT under relatively mild inflammation stimulation is stronger than the loss of PLT and megakarocyte against virus, but this growth advantage will be weakened with the aggravation of inflammation and infection. It has been confirmed in the literature that IL-6 has a similar role as thrombopenia in promoting the generation of PLT and regulating the proliferation and maturation of megakarocytic, but the specific mechanisms in vivo remain to be studied(Chen, et al 2017).

Clinical observation showed that thrombocytopenia may occur during the entire treatment process of COVID19, and the early changes of PLT have not been directly correlated with the severity of this disease, and the treatment outcome of COVID-19 with early PLT elevate and decrease remains to be further studied. Therefore, early PLT changes cannot be used as an indicator of COVID-19 severity. However, there are also many COVID-19 patients with severe and rapid PLT decline, which is still difficult to explain, resulting in the risk of bleeding complications. At present, the effect of drug therapy for increasing PLT remains to be observed, and active treatment of the primary disease should be one of the best treatment methods. To explore changes and mechanism of PLT after COVID-19 may be helpful for us to have a more comprehensive understanding of the occurrence, development, treatment and outcome of COVID-19 disease.

\section{Shortcomings and Acknowledgements}

Owing to the low positive rate of COVID-19 nucleic acid test, it is difficult to refine the treatment time before admission. However, the selected groups and treatments of the patients were basically homogeneous and comparable to some extent. The sample size is small, and the stability of personality index still needs to be supported by more samples.

Thanks for the support and help from the Cancer Center Affiliated to Tongji Medical College of Huazhong University of Science and Technology. Thank you for all contributions. This article is published by all authors and the unit agreed, There is no dispute.

\section{Funding and Role of the funding source}

Not applicable.

\section{Conflicts of interest}

No conflict of interest exists in the submission of this manuscript, and manuscript is approved by all authors for publication. I would like to declare on behalf of my co-authors that the work described was original research that has not been published previously, and not under consideration for publication elsewhere, in whole or in part. All the authors listed have approved the manuscript that encloses.

\section{Ethics approval and consent to participate}

Not applicable.

\section{Data Availability Statement}

Some or all data, models, or code generated or used during the study are available from the corresponding author by request.

\section{References}

Rommel MG E, Milde C, Eberle R, Schulze H, Modlich U(2019). Endothelial-platelet interactions in influenza-induced pneumonia: A potential therapeutic target.Anat Histol Embryol.49(5):606-619;DOI:

10.1111/ahe.12521

Huang C, Wang Y, Li X, Ren L, Zhao J, Hu Y, Zhang L, Fan G, Xu J, Gu X, Cheng Z, Yu T, Xia J, Wei Y, Wu W, Xie X, Yin W, Li H, Liu M, Xiao Y, Gao H, Guo L, Xie J, Wang G, Jiang R, Gao Z, Jin Q, Wang J, Cao B(2020). Clinical features of patients infected with 2019 novel coronavirus in Wuhan, China. Lancet. 15;395(10223):497-506. doi: 
10.1016/S0140-6736(20)30183-5.

Zhu L, Huang Z, Stalesen R, Hansson GK, Li N(2014). Platelets provoke distinct dynamics of immune responses by differentially regulating CD4+ T-cell proliferation. J Thromb Haemost. 12(7):1156-65. doi:

10.1111/jth.12612.

Yang M, Li CK, Li K, Hon KL, Ng MH, Chan PK,Fok TF. Hematological findings in SARS patients and possible mechanisms (review). Int J Mol Med. 14(2):311-5. PMID: 15254784.

Lefrancais E, Ortiz-Munoz G, Caudrillier A, Mallavia B, Liu F, Sayah DM, Thornton EE, Headley MB, David T, Coughlin SR, Krummel MF, Leavitt AD, Passegue E, Looney MR(2017). The lung is a site of platelet biogenesis and a reservoir for haematopoietic progenitors.Nature.7648(544):105-109.DOI: 10.1038/nature21706

Campbell RA, Schwertz H, Hottz ED, Rowley JW, Manne BK, Washington AV, Hunter-Mellado R, Tolley ND, Christensen M, Eustes AS, Montenont E, Bhatlekar S, Ventrone CH, Kirkpatrick BD, Pierce KK, Whitehead SS, Diehl SA, Bray PF, Zimmerman GA, Kosaka Y, Bozza PT, Bozza FA, Weyrich AS,Rondina MT(2019).Human megakaryocytes possess intrinsic antiviral immunity through regulated induction of IFITM3.Blood.;133(19):2013-2026.

DOI: 10.1182/blood-2018-09-873984

Chen,S.Su,YWang,J(2013).ROS-mediated platelet generation: a microenvironment-dependent manner for megakaryocyte proliferation, differentiation, and maturation[J].Cell Death Dis,4:e722.DOI:

10.1038/cddis.2013.253

\section{Hosted file}

Table.pdf available at https://authorea.com/users/372294/articles/490395-case-analysis-ofearly-covid-19-complicated-with-plt-abnormality 